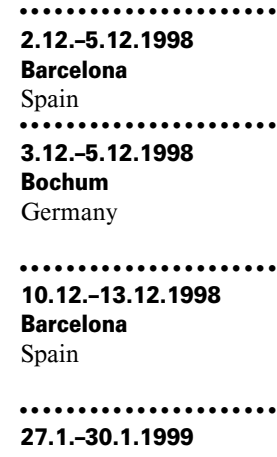

\section{3.-6.3. 1999}

Brussels

Belgium

\section{4.-11.4.1999 \\ Lugano \\ Switzerland \\ 24.4.-29.4.1999 San Diego, Calif., USA}

\section{Future sites:}

5.5.-10.5.2000

18.5.-23.5.2001

27.4.-1.5.2002

17.5.-21.5.2003

26.5.-29.5.1999

Istanbul

Turkey

\section{6.-25.6.1999}

Houston, Tex.

USA
Congreso Nacional de la Sociedad Española de Alergología e Immunología Clínica

Jahrestagung der Gesellschaft für Lungen- und Atmungsforschung

\section{World Asthma Meeting}

3ème Congrès de Pneumologie de Langue Française

\section{European Lung Cancer Working Party (20th Anniversary)}

14. Pneumologische Fortbildungsveranstaltung

\section{Kongress der Deutschen Gesellschaft für Pneumologie (DGP)/}

25. Kongress der Österreichischen Gesellschaft für Lungenerkrankungen und Tuberkulose (ÖGLUT)

\section{5th Postgraduate Course on Anaesthesiology}

American Thoracic Society International Congress

Toronto, Ont.

San Francisco, Calif.

Chicago, III.

Seattle, Wash.

Joint Meeting of the Paediatric Assembly, European Respiratory Society and European Society of Paediatric Allergy and Clinical Immunology

\section{Asthma 99}

1999 - International Conference on Asthma and Environmental Pollution
Information: Barcelona Convention Bureau, Tarragona 149-157, E-08015 Barcelona (Spain), Tel. +34 3423 1800, Fax +34 34232649

Information: BG Klinik Ber.................. Bürkle-de-la-Camp-Platz 1,

D-44789 Bochum (Germany),

Tel. +492343026753

General Information: AOPC, Edif. colon,

Av. Drassanes, 6, E-08001 Barcelona (Spain),

Tel. +34 330275 41, Fax +34 33011255 ,

E-Mail aopc@nexus.es

General Information: Pneumologie Développement,

Mme Chantal Anciaux,

41, Blvd St. Michel, F-75006 Paris (France),

Tel. +3314633 37 39, Fax +33146290110,

E-Mail pneumodev@magic.fr

Conference Secretariat: Mrs. Véronique Wanet,

Institut Jules Bordet, 1, rue Héger-Bordet,

B-1000 Brussels (Belgium),

Tel. +32 253535 22, Fax +32 253437 56,

E-Mail101473.1044@compuserve.com

Congress Secretariat: Brigitte Graf,

Medizinische Klinik I,

Friedrich-Alexander-Universität Erlangen-Nürnberg,

Krankenhausstr. 12,

D-91054 Erlangen (Deutschland)

Tel. +49 9131 853374, Fax +499131856327

Information:

DGP: Prof. Dr. D. Nolte, Städtisches Krankenhaus,

D-83435 Bad Reichenhall (Germany),

Tel. +49 8651772 401, Fax +49 8651772682

ÖGLUT: Prim. Dr. K. Aigner,

Krankenhaus der Elisabethinen,

A-4010 Linz (Austria),

Tel. +437327676 4205, Fax +437327676 2106

Organizing Secretariat: Alpha Studio,

Via Battisti 1, I-34125 Trieste (Italy),

Tel. +39 40 7600101, Fax +39 407600123 ,

E-Mail alpha@spin.it

Information: Deborah Richardt, Mgr. Meetings

Conferences, American Thoracic Society,

ALA/ATS International Conference, 1740 Broadway,

14th Floor, New York, NY 10019-4374 (USA),

Fax 212315 6498, E-Mail drichardt@lungusa.org

 Information: VIP Tourism, Cumhuriyet Cad. No. 269, Harbiye 80230 Istanbul (Turkey),

Fax +902122306425,

E-Mail ers.espaci@viptourism.com.tr

Information: Dr. V.M. Bhatnagar,

Alena Chemicals of Canada, P.O. Box 1779,

Cornwall, Ont. K6H 5 V7 (Canada),

Tel. 6139327702

\section{KARGER}

구 1998 S. Karger AG, Basel

Fax +41613061234 E-Mail karger@karger.ch www.karger.com
Accessible online at:

http://BioMedNet.com/karger 


\section{6.-25.6.1999}

Morschach ob

Brunnen/SZ

Switzerland

9.10.-13.10.1999

Madrid, Spain

Future sites:

30.8.-3.9.2000

14.10.-15.10.1999

Madras

India

\subsection{0.-20.10.1999}

Buenos Aires

Argentina

\subsubsection{9}

15.11.-20.11.1999

Trieste

Italy

\subsection{1.-21.11.1999}

Trieste

Italy
Jahrestagung der Schweizerischen Gesellschaft für Pneumologie

\section{Annual Congress of the European Respiratory Society (ERS)}

Florence, Italy

\section{Asthma 99}

1999 - International Conference on Asthma and Environmental Pollution

\section{XVlth World Congress of Asthmology}

\section{Pneumologische Fortbildungsveranstaltung}

APICE ‘99, 14th International Symposium on Critical Care Medicine

\section{8th Meeting of the Organ Failure Academy (OFA)}

Information: AKM AG, Clarastrasse 57,

CH-4058 Basel (Switzerland),

Tel. +416168677 11, Fax +41616867788,

E-Mailakm@bluewin.ch

Scientific Programme Coordination: ERS Paris Office, 60, rue de Vaugirard, F-75006 Paris (France),

Tel. +33145448584, Fax +33145443411,

E-Mail@ersnet.org

Information: Dr. V.M. Bhatnagar,

Alena Chemicals of Canada, P.O. Box 1779,

Cornwall, Ont. K6H 5V7 (Canada),

Tel. 6139327702

Congress Secretariat: Irigoyen Freyre 2670

3000 Santa Fe (Argentina),

Tel. + 5442 537638, Fax +54 42560773 ,

E-Mail interasm@neffen.satlink.net

Congress Secretariat: Brigitte Graf,

Medizinische Klinik I

Friedrich-Alexander-Universität Erlangen-Nürnberg,

Krankenhausstr. 12

D-91054 Erlangen (Deutschland)

Tel. +49 9131853374, Fax +49 9131856327

Organizing Secretariat: Alpha Studio,

Via Battisti 1, I-34125 Trieste (Italy),

Tel. +39 407600101, Fax +39 407600123 ,

E-Mailalpha@spin.it

Organizing Secretariat: Alpha Studio,

Via Battisti 1, I-34125 Trieste (Italy),

Tel. +3940 7600101, Fax +39 407600123 ,

E-Mail alpha@spin.it 